\title{
The Design Of Multiple Nodes Wireless Temperature Transmission System Based On STC15W1K24S And CC1101
}

\author{
Zhijian Yin ${ }^{1, a}$, Fan Wang ${ }^{2, b}$ and Kun Xiang ${ }^{3, c}$ \\ ${ }^{1}$ Jiangxi Science \& Technology Normal University, Nanchang, China \\ ${ }^{2}$ Jiangxi Science \& Technology Normal University, Nanchang, China \\ ${ }^{3}$ Jiangxi Science \& Technology Normal University, Nanchang, China \\ a86412656@qq.com, b1245905084@qq.com, c1244689313@qq.com
}

Keywords: CC1101; SPI; Temperature;

Abstract:With the development of science and technology, the wireless transmission system has a broad application field. This system need to collect the temperature data of two nodes. So we use the new single chip microcomputer STC15W1K24S as microprocessor, DS18B20 as temperature sensor, low power consumption wireless radio frequency transceiver CC1101 to transmit data collected from the two channels, and LCD1602 to display data. The single chip microcomputer has SPI interface, it can improve the efficiency of data transmission and has better real-time, when using its own SPI interface to communicate with CC1101. This system is battery-powered, it has small volume, low power consumption, high precision and easy to carry.

\section{The overall design and overview of system}

This system is multiple nodes wireless temperature transmission system, we will collect the temperature data of two channels and display it in the termination, realizing remote wireless transmission. The overall diagram of system design is divided into two parts of the acquisition node and terminal node.

Acquisition node consists of microprocessor, temperature sensor, power source and RF chip CC1101 as shown in Fig. 1.

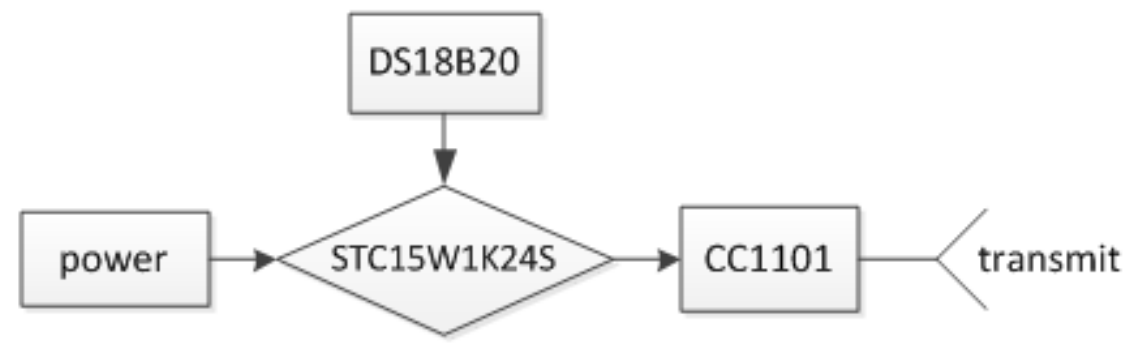

Fig. 1 The diagram of acquisition node

Terminal node is shown in Fig.2, including MCU, CC1101, power supply and display part.

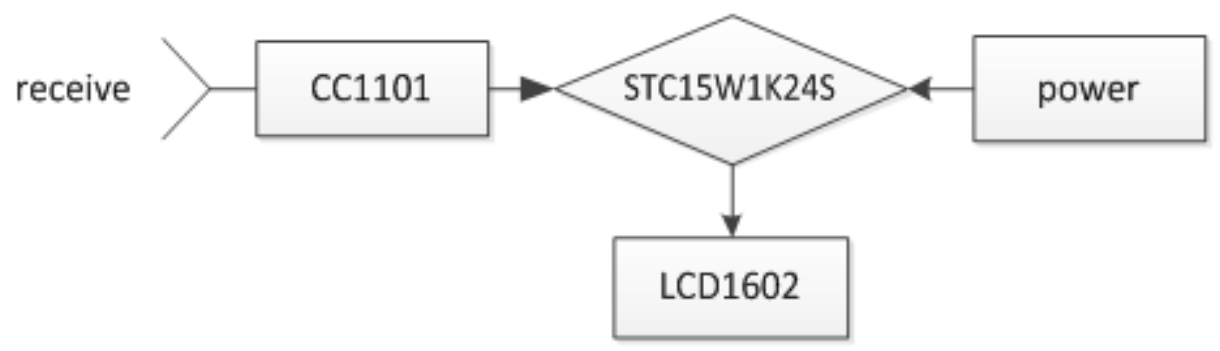

Fig. 2 The diagram of terminal node 


\section{Hardware design}

Single-chip microcomputer control and wireless module. STC15W1K24S is a single chip microcomputer of single clock/machine cycle (IT) produced by STC. It is a new generation of 8051 single chip microcomputer of wide voltage, high speed, high-reliable, low power consumption and strong anti-interference ${ }^{[1]}$, using the ninth generation of STC encryption technology, unable to decrypt. Its instruction code is fully compatible with the traditional 8051 , but 8 to 12 times faster. It integrates high precision clock $(\mathrm{R} / \mathrm{C})$ inside, $+1 \%$ temperature drift $\left(-40^{\circ} \mathrm{C} \sim+85^{\circ} \mathrm{C}\right),+0.6 \%$ temperature drift at normal temperature $\left(-20^{\circ} \mathrm{C} \sim+65^{\circ} \mathrm{C}\right)$. When ISP programming, $5 \mathrm{MHZ} 35 \mathrm{MHZ}$ wide range can be set up. External crystals and external reset circuit can be completely eliminated, it can fully meet requirements of small volume, low cost, low power consumption, high precision and strong anti-interference. It has one set of high-speed synchronous serial communication port (SPI), will improve data transmission rate when communicating with CC1101.

The RF module CC1101 is the latest high-performance wireless communication chip of Chipcon company, its circuit mainly set in $315 \mathrm{MHz}, 433 \mathrm{MHz}, 868 \mathrm{MHz}$ and $915 \mathrm{MHz}$ of ISM (industrial, scientific and medical) and SRD (short range device) frequency band, also can easily set to $300 \sim$ $348 \mathrm{MHz}, 400 \sim 464 \mathrm{MHz}$ and $800 \sim 928 \mathrm{MHz}$ frequency ${ }^{[2]}$. Working voltage is $3.3 \mathrm{~V}$, maximum transmission rate can be $500 \mathrm{~KB} / \mathrm{s}$, penetrability is better in the process of transmission, transmission distance can reach $300 \sim 500 \mathrm{~m}$ in the open place. It has the function of wireless wake-up, high sensitivity, strong anti-interference and low power consumption, can be widely applied to various occasions of short distance wireless communication ${ }^{[3]}$. The connection of $\mathrm{CC} 1101$ and STC15W1K24S with SPI is shown in Fig.3, P1.3, P1.4, P1.5 each connect to SI, SO, SCLK of $\mathrm{CC} 1101$, working frequency is $433 \mathrm{MHZ}$, the temperature collected by MCU is sent to CC1101 for transmitting, in the terminal node, the data receiving by $\mathrm{CC} 1101$ is sent to $\mathrm{MCU}$ for processing displaying.

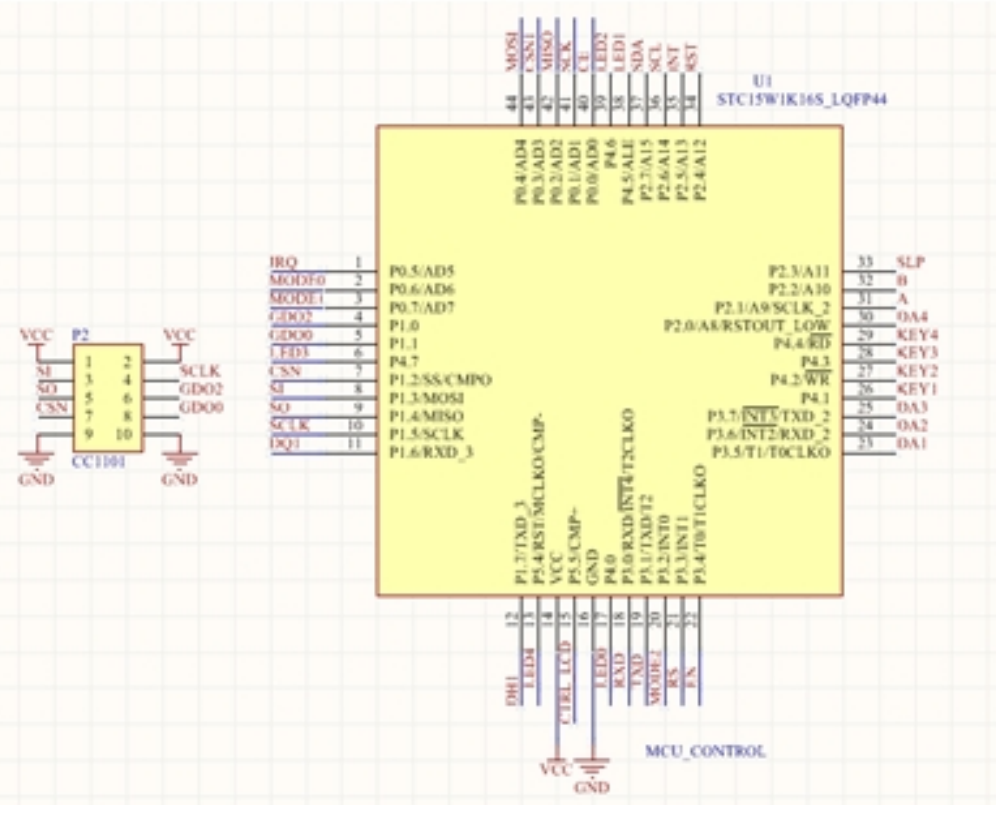

Fig.3 The circuit of MCU and CC1101

The temperature acquisition module. This system has the characteristics of small volume, low power consumption and simple circuit, so there is a demand for the selection of the temperature sensor. Based on the above requirements, temperature measurement is one wire digital temperature sensor (DS18B20) produced by DALLAS company[4], it is small in size, low prices, and has 3 pin T0-92 small packaging format. The scope of its power supply is $3 \sim 5.5 \mathrm{~V}$, it can measure the temperature of $-55 \sim+125^{\circ} \mathrm{C}$. Circuit is shown in Fig.4. 


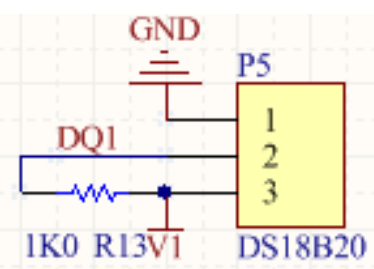

Fig.4 Temperature collection circuit

Display module. Display part adopts the four wire drive connection of LCD1602 shown in Fig.5, only need four high data port of 1602 connecting with the IO port of MCU, this method can greatly save IO ports. RS and EN is controlled by two IO ports of STC15W1K24S, R/W is grounding, this kind of connection only can write instructions and data, but cannot read data from the LCD.

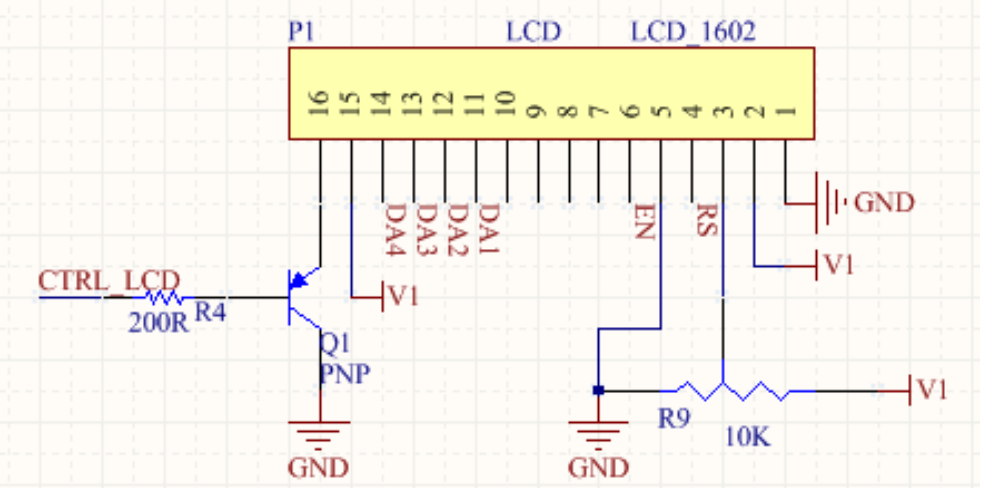

Fig. 5 LCD1602 displaying circuit

Power supply module. As shown in table 1, the working voltage of each device is different used in this system.

Table 1: Working voltage of devices

\begin{tabular}{|c|c|}
\hline devices & Working voltage $(\mathbf{V})$ \\
\hline STC15W1K24S & $2.6 \sim 5.5$ \\
\hline CC1101 & $1.8 \sim 3.6$ \\
\hline LCD1602 & $4.5 \sim 5.5$ \\
\hline DS18B20 & $3.0 \sim 5.5$ \\
\hline
\end{tabular}

The power of this system is provided by three dry batteries, its voltage is about $4.5 \mathrm{~V}$. Because the working voltage of CC1101 is less than $3.6 \mathrm{~V}$, so we need to use LM117 chips to transform it into 3.3 $\mathrm{V}$ and three capacitors to filter. The circuit is shown in fig. 6

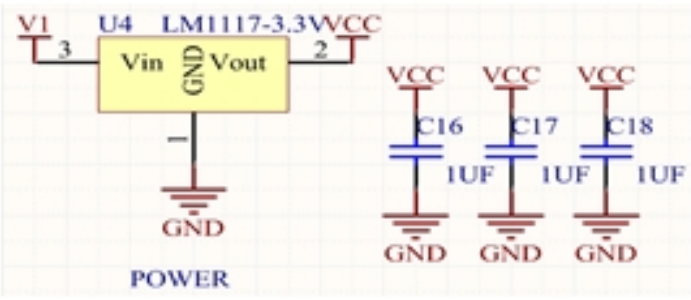

Fig.6 The power circuit

\section{Software design}

The design of main program. The main program flow chart of collecting node is shown in fig.7. There is a while loop in the main program, it will read and send the temperature data continually. 
As shown in fig.8, it is need to receive the data of the two different nodes, so it should use the channel 1 and channel 2 of CC1101. The first node uses the channel 1,and the data displays in the first line of the LCD1602, oppositely, node 2 is for channel 2 and data displays in line 2.

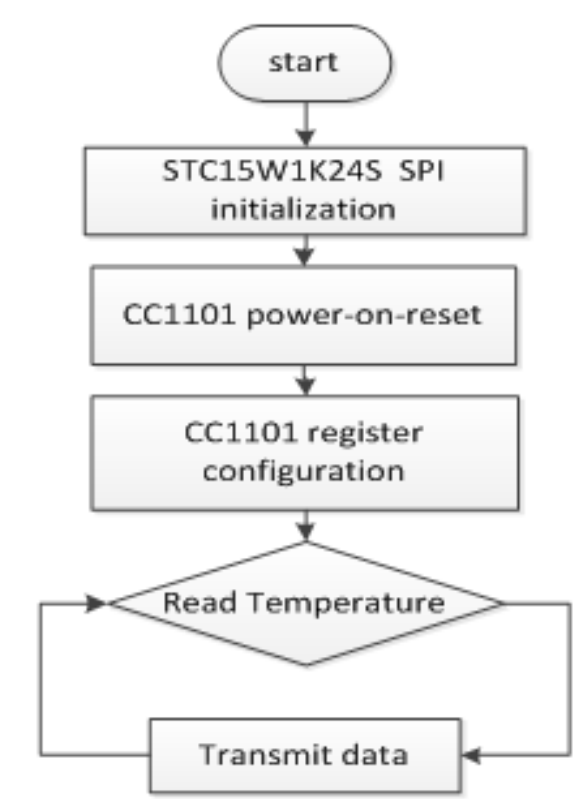

Fig.7 The main program flow chart of collecting node

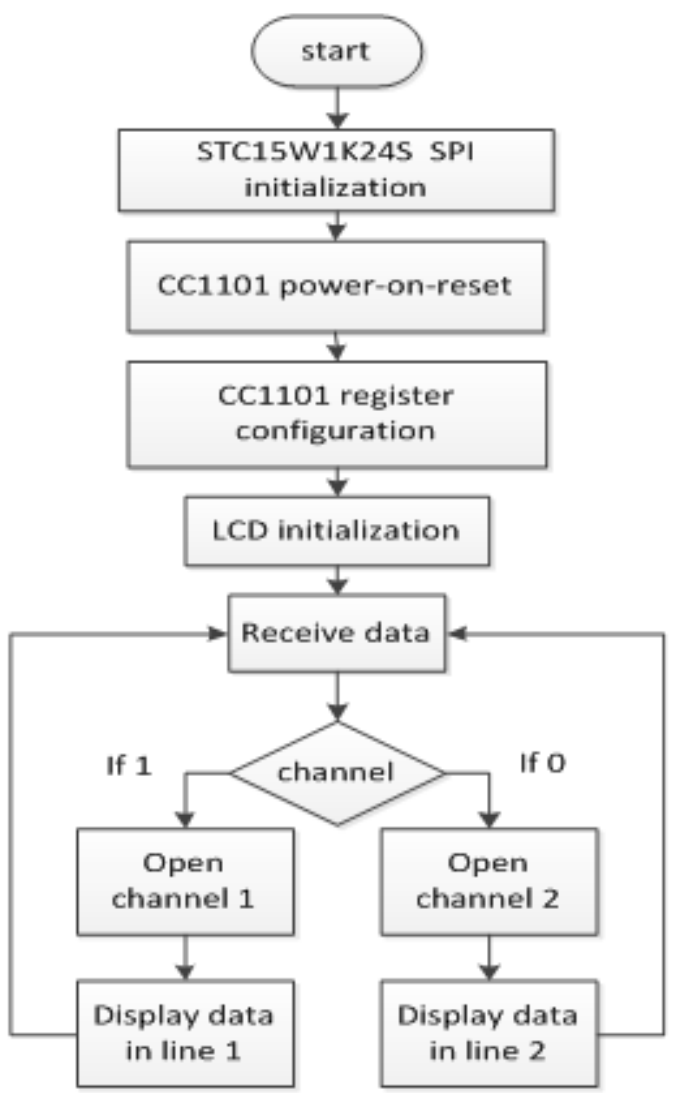

Fig.8 The main program flow chart of terminal node

SPI initialization and registers configuration of CC1101. The SPI interface of STC15W1K24S is a full-duplex, high speed, synchronous communication bus, there are two kinds of operating modes: the master mode and the slave mode. The main function register MCU SPI interface is SPCTL (SPI control register), SPSTAT (SPI status register) and SPDAT (SPI data registers). SPI initialization need to initialize the three register. SPSTAT need to write "1" to the flags(SPIF and WCOL) for clearing. In this system, the collecting and terminal node all use the main mode, it need to will write "1" to SPCTL SSIG, MSTR and SPEN in order to set the master mode.

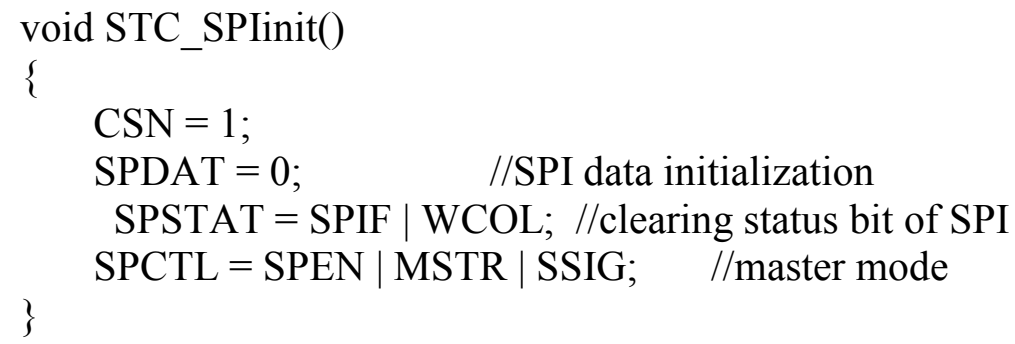

Because it is the use of single chip microcomputer hardware SPI interface communicating with CC1101, instead of using software to simulate SPI, it will involve the register SPSTAT and SPDAT of MCU. The function(Spi_RW) will realize sending a byte from SPI interface, halSpiWriteReg is used to configure the written register of CC1101. CC1101 has more than 40 registers to be configured, there are many kinds of working mode, the configuration of other registers can consult the CC1101 data handbook and the subroutines followed. 


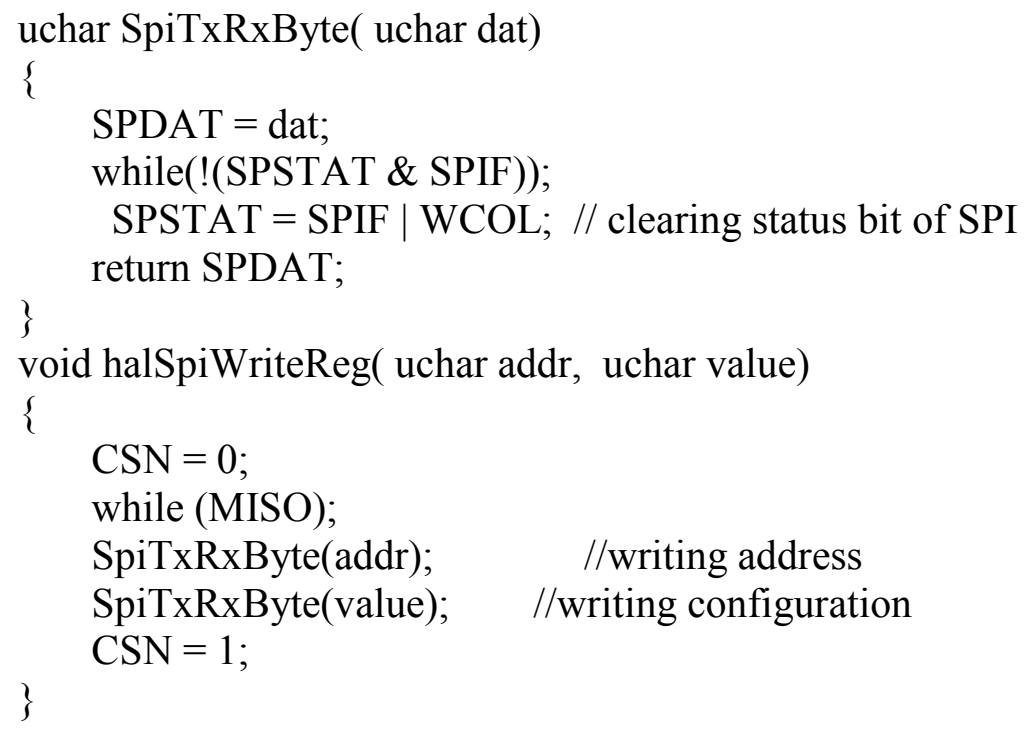

\section{System debugging and conclusion}

Put two acquisition nodes in a classroom of upstairs and downstairs of my laboratory, put the terminal node in our lab, test on the same day when the weather is sunny and the temperature is higher. The acquisition node downstairs use the channel 1 of CC1101, upstairs is channel 2, the data receiving by terminal node is shown in fig. 9 , it is $28.9^{\circ} \mathrm{C}$ and $28.8^{\circ} \mathrm{C}$.

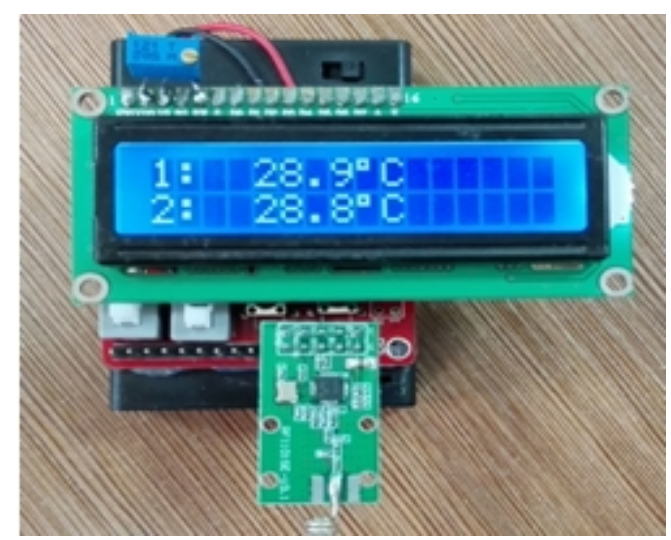

Fig. 9 Data displaying in terminal node

\section{Reference}

[1] STC15 series microcontroller device manual[Z]. Hong Jing technology company, 2015

[2] Wei Chen, Guoliang Shi. The design of workshop wireless communication system based on CC1101[J]. Communication technology, 2011,44(12):42-44,47.

[3]LIU Yu,ZHAO Jian-ping,LIU Na,HOU Li-jing.Design on Real-time Health Monitoring Detectio n System Based on MSP430 and CC1101[J].Communications Technology,2012,12(45):127-130.

[4] Shujuan Chai. The design of wireless monitoring alarm system for home-bound elders[D]. Qufu: Qufu Normal University, 2010.

[5] LijunLi, DaihuaWang, JingZu. The design of wireless data transmission system based on CC1100[J]. Foreign Electronic Measurement Technology, 2007, 26(12): 42-45

[6] Xiaolong $\mathrm{Lu}$. The design of the intelligent greenhouse monitoring system based on CC1101. Master's thesis of Donghua University. 2013: 30-35. 